\title{
JENSEN'S OPERATOR INEQUALITY AND ITS CONVERSES
}

\author{
FRANK HANSEN, JOSIP PEČARIĆ and IVAN PERIĆ
}

(Dedicated to the memory of Gert K. Pedersen)

\begin{abstract}
We give a general formulation of Jensen's operator inequality for unital fields of positive linear mappings, and we consider different types of converse inequalities.
\end{abstract}

\section{Introduction}

Let $I$ be a real interval of any type. A continuous function $f: I \rightarrow \mathrm{R}$ is said to be operator convex if

$$
f(\lambda x+(1-\lambda) y) \leq \lambda f(x)+(1-\lambda) f(y)
$$

holds for each $\lambda \in[0,1]$ and every pair of self-adjoint operators $x$ and $y$ (acting) on an infinite dimensional Hilbert space $H$ with spectra in $I$ (the ordering is defined by setting $x \leq y$ if $y-x$ is positive semi-definite).

Let $f$ be an operator convex function defined on an interval $I$. Ch. Davis [4] proved $^{1}$ a Schwarz inequality

$$
f(\phi(x)) \leq \phi(f(x)),
$$

where $\phi: \mathscr{A} \rightarrow B(H)$ is a unital completely positive linear map from a $C^{*}$ algebra $\mathscr{A}$ to linear operators on a Hilbert space $H$, and $x$ is a self-adjoint element in $\mathscr{A}$ with spectrum in $I$. Subsequently M. D. Choi [3] noted that it is enough to assume that $\phi$ is unital and positive. In fact, the restriction of $\phi$ to the commutative $C^{*}$-algebra generated by $x$ is automatically completely positive by a theorem of Stinespring.

\footnotetext{
Received March 14, 2006.

${ }^{1}$ There is small typo in the proof. Davis states that $\phi$ by Stinespring's theorem can be written on the form $\phi(x)=P \rho(x) P$ where $\rho$ is a $*$-homomorphism to $B(H)$ and $P$ is a projection acting on $H$. In fact, $H$ may be embedded in a Hilbert space $K$ on which $\rho$ and $P$ acts. The theorem then follows by the calculation $f(\phi(x))=f(P \rho(x) P) \leq P f(\rho(x)) P=P \rho(f(x)) P=\phi(f(x))$, where the pinching inequality, proved by Davis in the same paper, is applied.
} 
F. Hansen and G. K. Pedersen [7] proved a Jensen type inequality

$$
f\left(\sum_{i=1}^{n} a_{i}^{*} x_{i} a_{i}\right) \leq \sum_{i=1}^{n} a_{i}^{*} f\left(x_{i}\right) a_{i}
$$

for operator convex functions $f$ defined on an interval $I=[0, \alpha)$ (with $\alpha \leq \infty$ and $f(0) \leq 0)$ and self-adjoint operators $x_{1}, \ldots, x_{n}$ with spectra in $I$ assuming that $\sum_{i=1}^{n} a_{i}^{*} a_{i}=\mathbf{1}$. The restriction on the interval and the requirement $f(0) \leq$ 0 was subsequently removed by B. Mond and J. Pečarić in [13], cf. also [8].

The inequality (3) is in fact just a reformulation of (2) although this was not noticed at the time. It is nevertheless important to note that the proof given in [7] and thus the statement of the theorem, when restricted to $n \times n$ matrices, holds for the much richer class of $2 n \times 2 n$ matrix convex functions. Hansen and Pedersen used (3) to obtain elementary operations on functions, which leave invariant the class of operator monotone functions. These results then served as the basis for a new proof of Löwner's theorem applying convexity theory and Krein-Milman's theorem.

Finally B. Mond and J. Pečarić [12] proved the inequality

$$
f\left(\sum_{i=1}^{n} w_{i} \phi_{i}\left(x_{i}\right)\right) \leq \sum_{i=1}^{n} w_{i} \phi_{i}\left(f\left(x_{i}\right)\right)
$$

for operator convex functions $f$ defined on an interval $I$, where $\phi_{i}: B(H) \rightarrow$ $B(K)$ are unital positive linear maps, $x_{1}, \ldots, x_{n}$ are self-adjoint operators with spectra in $I$ and $w_{1}, \ldots, w_{n}$ are non-negative real numbers with sum one.

The aim of this paper is to find an inequality which contains (3), (2) and (4) as special cases. Since the inequality in (4) was the motivating step for obtaining converses of Jensen's inequality using the so called Mond-Pečarić method, we also give some results pertaining to converse inequalities in the new formulation.

\section{The main result}

\section{Continuous Fields of Operators}

Let $T$ be a locally compact Hausdorff space and let $\mathscr{A}$ be a $C^{*}$-algebra. We say that a field $\left(x_{t}\right)_{t \in T}$ of operators in $\mathscr{A}$ is continuous if the function $t \rightarrow x_{t}$ is norm continuous on $T$. If in addition $\mu$ is a Radon measure on $T$ and the function $t \rightarrow\left\|x_{t}\right\|$ is integrable, then we can form the Bochner integral $\int_{T} x_{t} d \mu(t)$, which is the unique element in $\mathscr{A}$ such that

$$
\varphi\left(\int_{T} x_{t} d \mu(t)\right)=\int_{T} \varphi\left(x_{t}\right) d \mu(t)
$$


for every linear functional $\varphi$ in the norm dual $\mathscr{A}^{*}$, cf. [8, Section 4.1].

Assume furthermore that there is a field $\left(\phi_{t}\right)_{t \in T}$ of positive linear mappings $\phi_{t}: \mathscr{A} \rightarrow \mathscr{B}$ from $\mathscr{A}$ to another $C^{*}$-algebra $\mathscr{B}$. We say that such a field is continuous if the function $t \rightarrow \phi_{t}(x)$ is continuous for every $x \in \mathscr{A}$. If the $C^{*}$-algebras are unital and the field $t \rightarrow \phi_{t}(\mathbf{1})$ is integrable with integral $\mathbf{1}$, we say that $\left(\phi_{t}\right)_{t \in T}$ is unital.

THEOREM 2.1. Let $f: I \rightarrow \mathrm{R}$ be an operator convex function defined on an interval I, and let $\mathscr{A}$ and $\mathscr{B}$ be unital $C^{*}$-algebras. If $\left(\phi_{t}\right)_{t \in T}$ is a unital field of positive linear mappings $\phi_{t}: \mathscr{A} \rightarrow \mathscr{B}$ defined on a locally compact Hausdorff space $T$ with a bounded Radon measure $\mu$, then the inequality

$$
f\left(\int_{T} \phi_{t}\left(x_{t}\right) d \mu(t)\right) \leq \int_{T} \phi_{t}\left(f\left(x_{t}\right)\right) d \mu(t)
$$

holds for every bounded continuous field $\left(x_{t}\right)_{t \in T}$ of self-adjoint elements in $\mathscr{A}$ with spectra contained in I.

Proof. We first note that the function $t \rightarrow \phi_{t}\left(x_{t}\right) \in \mathscr{B}$ is continuous and bounded, hence integrable with respect to the bounded Radon measure $\mu$. We may organize the set $C B(T, \mathscr{A})$ of bounded continuous functions on $T$ with values in $\mathscr{A}$ as a normed involutive algebra by applying the point-wise operations and setting

$$
\left\|\left(y_{t}\right)_{t \in T}\right\|=\sup _{t \in T}\left\|y_{t}\right\|, \quad\left(y_{t}\right)_{t \in T} \in C B(T, \mathscr{A}),
$$

and it is not difficult to verify that the norm is already complete and satisfy the $C^{*}$-identity. In fact, this is a standard construction in $C^{*}$-algebra theory. It follows that $f\left(\left(x_{t}\right)_{t \in T}\right)=\left(f\left(x_{t}\right)\right)_{t \in T}$. We then consider the mapping

$$
\pi: C B(T, \mathscr{A}) \rightarrow \mathscr{B}
$$

defined by setting

$$
\pi\left(\left(x_{t}\right)_{t \in T}\right)=\int_{T} \phi_{t}\left(x_{t}\right) d \mu(t)
$$

and note that it is a unital positive linear map. Setting $x=\left(x_{t}\right)_{t \in T} \in C B(T, \mathscr{A})$, we use inequality (2) to obtain

$$
f\left(\pi\left(\left(x_{t}\right)_{t \in T}\right)\right)=f(\pi(x)) \leq \pi(f(x))=\pi\left(f\left(\left(x_{t}\right)_{t \in T}\right)\right)=\pi\left(\left(f\left(x_{t}\right)\right)_{t \in T}\right),
$$

which is the statement of the theorem.

To illustrate various techniques in proving Jensen's operator inequality, we give two proofs of Theorem 2.1 in the discrete case $T=\{1, \ldots, n\}$. 
Proof. By using the continuity of $f$ and uniform approximation of selfadjoint operators by simple operators, we may assume that $x_{i}$ has a spectral resolution on the form

$$
x_{i}=\sum_{j \in J_{i}} t_{i, j} e_{i, j}, \quad i=1, \ldots, n,
$$

where each $J_{i}$ is a finite set and $\sum_{j \in J_{i}} e_{i, j}=\mathbf{1}$. We then have

$$
\begin{aligned}
f\left(\sum_{i=1}^{n} \phi_{i}\left(x_{i}\right)\right) & =f\left(\sum_{i=1}^{n} \phi_{i}\left(\sum_{j \in J_{i}} t_{i, j} e_{i, j}\right)\right)=f\left(\sum_{i=1}^{n} \sum_{j \in J_{i}} t_{i, j} \phi_{i}\left(e_{i, j}\right)\right) \\
& =f\left(\sum_{i=1}^{n} \sum_{j \in J_{i}} \sqrt{\phi_{i}\left(e_{i, j}\right)} t_{i, j} \sqrt{\phi_{i}\left(e_{i, j}\right)}\right) \\
& \leq \sum_{i=1}^{n} \sum_{j \in J_{i}} \sqrt{\phi_{i}\left(e_{i, j}\right)} f\left(t_{i, j}\right) \sqrt{\phi_{i}\left(e_{i, j}\right)} \\
& =\sum_{i=1}^{n} \phi_{i}\left(\sum_{j \in J_{i}} f\left(t_{i, j}\right) e_{i, j}\right)=\sum_{i=1}^{n} \phi_{i}\left(f\left(x_{i}\right)\right) .
\end{aligned}
$$

THE SECOND PROOF. We use an the idea from [9], confer also [13]. If $f$ is operator convex in $I=[0,1)$ and $f(0) \leq 0$, then there is a connection $\sigma$ such that $-f(t)=t \sigma(1-t)$. We use the following two properties of a connection, cf. [1], [9].

(1) $\phi(a \sigma b) \leq \phi(a) \sigma \phi(b)$ for positive linear maps $\phi$ and positive operators $a$ and $b$.

(2) $\sum_{i=1}^{n} a_{i} \sigma b_{i} \leq\left(\sum_{i=1}^{n} a_{i}\right) \sigma\left(\sum_{i=1}^{n} b_{i}\right)$ for positive $n$-tuples $\left(a_{1}, \ldots, a_{n}\right)$ and $\left(b_{1}, \ldots, b_{n}\right)$. (subadditivity)

We then obtain

$$
\begin{aligned}
-\sum_{i=1}^{n} \phi_{i}\left(f\left(x_{i}\right)\right) & =\sum_{i=1}^{n} \phi_{i}\left(x_{i} \sigma\left(\mathbf{1}-x_{i}\right)\right) \leq \sum_{i=1}^{n} \phi_{i}\left(x_{i}\right) \sigma \phi_{i}\left(\mathbf{1}-x_{i}\right) \\
& \leq\left(\sum_{i=1}^{n} \phi_{i}\left(x_{i}\right)\right) \sigma\left(\sum_{i=1}^{n} \phi_{i}\left(\mathbf{1}-x_{i}\right)\right) \\
& =\left(\sum_{i=1}^{n} \phi_{i}\left(x_{i}\right)\right) \sigma\left(\mathbf{1}-\sum_{i=1}^{n} \phi_{i}\left(x_{i}\right)\right)=-f\left(\sum_{i=1}^{n} \phi_{i}\left(x_{i}\right)\right) .
\end{aligned}
$$


Consider now an arbitrary operator convex function $f$ defined on $[0,1)$. The function $\tilde{f}(x)=f(x)-f(0)$ satisfies $\tilde{f}(0)=0$ hence

$$
f\left(\sum_{i=1}^{n} \phi_{i}\left(x_{i}\right)\right)-f(0) \mathbf{1} \leq \sum_{i=1}^{n} \phi_{i}\left(f\left(x_{i}\right)\right)-f(0) \sum_{i=1}^{n} \phi_{i}(\mathbf{1})
$$

from which the statement follows. We finally obtain (5) in this setting for a function $f$ defined on an arbitrary interval $[\alpha, \beta)$ by considering the function $g(x)=f((\beta-\alpha) x+\alpha)$ on the interval $[0,1)$.

Inequality (5) is obviously a generalization of the inequalities (3), (2) and (4).

\section{Converses of Jensen's inequality}

The following theorem should be compared with Theorem 2.3 in [10]. For a function $f:[m, M] \rightarrow \mathbf{R}$ we use the standard notation:

$$
\alpha_{f}=\frac{f(M)-f(m)}{M-m} \quad \text { and } \quad \beta_{f}=\frac{M f(m)-m f(M)}{M-m} .
$$

We will apply functions $F(t, s)$ of two real variables to operators. In simple cases, when it makes sense, we may just replace numbers with operators. If for example $F(t, s)=s^{-1} t^{1 / 2} s^{-1}$, we may set $F(x, y)=y^{-1} x^{1 / 2} y^{-1}$. Otherwise we may use the functional calculus on tensor products, see for example [6].

THEOREM 3.1. Let $\left(x_{t}\right)_{t \in T}$ be a bounded continuous field of self-adjoint elements in a unital $C^{*}$-algebra $\mathscr{A}$ with spectra in $[m, M]$ defined on a locally compact Hausdorff space T equipped with a Radon measure $\mu$, and let $\left(\phi_{t}\right)_{t \in T}$ be a unital field of positive linear maps $\phi_{t}: \mathscr{A} \rightarrow \mathscr{B}$ from $\mathscr{A}$ to another unital $C^{*}$-algebra $\mathscr{B}$. Let $f, g:[m, M] \rightarrow \mathrm{R}$ and $F: U \times V \rightarrow \mathrm{R}$ be functions such that $f([m, M]) \subset U, g([m, M]) \subset V$ and $F$ is bounded. If $F$ is operator monotone in the first variable and $f$ is convex in the interval $[m, M]$, then

$$
\begin{aligned}
F\left[\int_{T} \phi_{t}\left(f\left(x_{t}\right)\right) d \mu(t), g\left(\int_{T} \phi_{t}\left(x_{t}\right) d \mu(t)\right)\right] & \\
& \leq \sup _{m \leq z \leq M} F\left[\alpha_{f} z+\beta_{f}, g(z)\right] \mathbf{1} .
\end{aligned}
$$

In the dual case (when $f$ is concave) the opposite inequality holds in (7) with inf instead of sup. 
Proof. For convex $f$ the inequality $f(z) \leq \alpha_{f} z+\beta_{f}$ holds for every $z \in[m, M]$. Thus, by using functional calculus, $f\left(x_{t}\right) \leq \alpha_{f} x_{t}+\beta_{f} \mathbf{1}$ for every $t \in T$. Applying the positive linear maps $\phi_{t}$ and integrating, we obtain

$$
\int_{T} \phi_{t}\left(f\left(x_{t}\right)\right) d \mu(t) \leq \alpha_{f} \int_{T} \phi_{t}\left(x_{t}\right) d \mu(t)+\beta_{f} \mathbf{1} .
$$

Now, using operator monotonicity of $F(\cdot, v)$, we obtain

$$
\begin{aligned}
& F\left[\int_{T} \phi_{t}\left(f\left(x_{t}\right)\right) d \mu(t), g\left(\int_{T} \phi_{t}\left(x_{t}\right) d \mu(t)\right)\right] \\
& \quad \leq F\left[\alpha_{f} \int_{T} \phi_{t}\left(x_{t}\right) d \mu(t)+\beta_{f} \mathbf{1}, g\left(\int_{T} \phi_{t}\left(x_{t}\right) d \mu(t)\right)\right] \\
& \quad \leq \sup _{m \leq z \leq M} F\left[\alpha_{f} z+\beta_{f}, g(z)\right] \mathbf{1} .
\end{aligned}
$$

Numerous applications of the previous theorem can be given (see [10]). We give generalizations of some results from [14].

THEOREM 3.2. Let $\left(A_{t}\right)_{t \in T}$ be a continuous field of positive operators on a Hilbert space $H$ defined on a locally compact Hausdorff space T equipped with a Radon measure $\mu$. We assume the spectra are in $[m, M]$ for some $0<m<M$. Let furthermore $\left(x_{t}\right)_{t \in T}$ be a continuous field of vectors in $H$ such that $\int_{T}\left\|x_{t}\right\|^{2} d \mu(t)=1$. Then for any $\lambda \geq 0, p \geq 1$ and $q \geq 1$ we have

$$
\left(\int_{T}\left\langle A_{t}^{p} x_{t}, x_{t}\right\rangle d \mu(t)\right)^{1 / q}-\lambda \int_{T}\left\langle A_{t} x_{t}, x_{t}\right\rangle d \mu(t) \leq C(\lambda, m, M, p, q),
$$

where the constant

$$
C(\lambda, m, M, p, q)= \begin{cases}M\left(M^{\frac{p}{q}-1}-\lambda\right), & 0<\lambda \leq \frac{\alpha_{p}}{q} M^{p\left(\frac{1}{q}-1\right)} \\ \frac{q-1}{q}\left(\frac{q}{\alpha_{p}} \lambda\right)^{\frac{1}{1-q}}+\frac{\beta_{p}}{\alpha_{p}} \lambda, & \frac{\alpha_{p}}{q} M^{p\left(\frac{1}{q}-1\right)} \leq \lambda \leq \frac{\alpha_{p}}{q} m^{p\left(\frac{1}{q}-1\right)} \\ m\left(m^{\frac{p}{q}-1}-\lambda\right), & \frac{\alpha_{p}}{q} m^{p\left(\frac{1}{q}-1\right)} \leq \lambda\end{cases}
$$

and $\alpha_{p}$ and $\beta_{p}$ are the constants $\alpha_{f}$ and $\beta_{f}$ associated with the function $f(z)=$ $z^{p}$. 
Proof. Applying Theorem 3.1 for the functions

$$
f(z)=z^{p}, \quad F(u, v)=u^{1 / q}-\lambda v,
$$

and unital fields of positive linear maps $\phi_{t}: B(H) \rightarrow \mathbf{C}$ defined by setting $\phi_{t}(A)=\left\langle A x_{t}, x_{t}\right\rangle$ for $t \in T$, the problem is reduced to determine $\sup _{m \leq z \leq M} H(z)$ where $H(z)=\left(\alpha_{p} z+\beta_{p}\right)^{1 / q}-\lambda z$.

The following Corollary is a generalization of Theorem 5 in [14]. The $r$ geometric mean $A \#_{r} B$ introduced by F. Kubo and T. Ando in [11] is defined by setting

$$
A \#_{r} B=A^{1 / 2}\left(A^{-1 / 2} B A^{-1 / 2}\right)^{r} A^{1 / 2}
$$

for positive invertible operators $A$ and $B$.

Corollary 3.3. Let $\left(A_{t}\right)_{t \in T}$ and $\left(B_{t}\right)_{t \in T}$ be continuous fields of positive invertible operators on a Hilbert space $H$ defined on a locally compact Hausdorff space T equipped with a Radon measure $\mu$ such that

$$
m_{1} \mathbf{1} \leq A_{t} \leq M_{1} \mathbf{1} \quad \text { and } \quad m_{2} \mathbf{1} \leq B_{t} \leq M_{2} \mathbf{1}
$$

for all $t \in T$ for some $0<m_{1}<M_{1}$ and $0<m_{2}<M_{2}$. Then for any $\lambda \geq 0, s \geq 1, p \geq 1$ and any continuous field $\left(x_{t}\right)_{t \in T}$ of vectors in $H$ such that $\int_{T}\left\|x_{t}\right\|^{2} d \mu(t)=1$ we have

$$
\begin{aligned}
& \left(\int_{T}\left\langle A_{t}^{p} x_{t}, x_{t}\right\rangle d \mu(t)\right)^{1 / p}\left(\int_{T}\left\langle B_{t}^{q} x_{t}, x_{t}\right\rangle d \mu(t)\right)^{1 / q} \\
& \quad-\lambda \int_{T}\left\langle B_{t}^{q} \#_{1 / s} A_{t}^{p} x_{t}, x_{t}\right\rangle d \mu(t) \leq C\left(\lambda, \frac{m_{1}^{p / s}}{M_{2}^{q / s}}, \frac{M_{1}^{p / s}}{m_{2}^{q / s}}, s, p\right) M_{2}^{q}
\end{aligned}
$$

where the constant $C$ is defined in Theorem 3.2 and $1 / p+1 / q=1$.

Proof. By using Theorem 3.2 we obtain for any $\lambda \geq 0$, for any continuous field $\left(C_{t}\right)_{t \in T}$ of positive operators with $m \mathbf{1} \leq C_{t} \leq M \mathbf{1}$ and a square integrable continuous field $\left(y_{t}\right)_{t \in T}$ of vectors in $H$ the inequality

$$
\begin{aligned}
& \left(\int_{T}\left\langle C_{t}^{s} y_{t}, y_{t}\right\rangle d \mu(t)\right)^{1 / p}\left(\int_{T}\left\langle y_{t}, y_{t}\right\rangle d \mu(t)\right)^{1 / q} \\
& \quad-\lambda \int_{T}\left\langle C_{t} y_{t}, y_{t}\right\rangle d \mu(t) \leq C(\lambda, m, M, s, p) \int_{T}\left\langle y_{t}, y_{t}\right\rangle d \mu(t) .
\end{aligned}
$$

Set now $C_{t}=\left(B_{t}^{-q / 2} A_{t}^{p} B_{t}^{-q / 2}\right)^{1 / s}$ and $y_{t}=B_{t}^{q / 2} x_{t}$ for $t \in T$ in (11) and observe that

$$
\frac{m_{1}^{p / s}}{M_{2}^{q / s}} \mathbf{1} \leq\left(B_{t}^{-q / 2} A_{t}^{p} B_{t}^{-q / 2}\right)^{1 / s} \leq \frac{M_{1}^{p / s}}{m_{2}^{q / s}} \mathbf{1} .
$$


By using the definition of the $1 / s$-geometric mean and rearranging (11) we obtain

$$
\begin{gathered}
\left(\int_{T}\left\langle A_{t}^{p} x_{t}, x_{t}\right\rangle d \mu(t)\right)^{1 / p}\left(\int_{T}\left\langle B_{t}^{q} x_{t}, x_{t}\right\rangle d \mu(t)\right)^{1 / q} \\
\quad-\lambda \int_{T}\left\langle B_{t}^{q} \#_{1 / s} A_{t}^{p} x_{t}, x_{t}\right\rangle d \mu(t) \\
\leq C\left(\lambda, \frac{m_{1}^{p / s}}{M_{2}^{q / s}}, \frac{M_{1}^{p / s}}{m_{2}^{q / s}}, s, p\right) \int_{T}\left\langle B_{t}^{q} x_{t}, x_{t}\right\rangle d \mu(t) \\
\leq C\left(\lambda, \frac{m_{1}^{p / s}}{M_{2}^{q / s}}, \frac{M_{1}^{p / s}}{m_{2}^{q / s}}, s, p\right) M_{2}^{q}
\end{gathered}
$$

which gives (10).

In the present context we may obtain results of the Li-Mathias type by using Theorem 3.1 and the following result which is a simple consequence of Theorem 2.1.

THEOREM 3.4. Let $\left(x_{t}\right)_{t \in T}$ be a bounded continuous field of self-adjoint elements in a unital $C^{*}$-algebra $\mathscr{A}$ defined on a locally compact Hausdorff space $T$ equipped with a Radon measure $\mu$. We assume the spectra are in $[m, M]$. Let furthermore $\left(\phi_{t}\right)_{t \in T}$ be a unital field of positive linear maps $\phi_{t}$ : $\mathscr{A} \rightarrow \mathscr{B}$ from $\mathscr{A}$ to another unital $C^{*}$-algebra $\mathscr{B}$. Let $f, g:[m, M] \rightarrow \mathrm{R}$ and $F: U \times V \rightarrow \mathrm{R}$ be functions such that $f([m, M]) \subset U, g([m, M]) \subset V$ and $F$ is bounded. If $F$ is operator monotone in the first variable and $f$ is operator convex in the interval $[m, M]$, then

$$
\begin{aligned}
F\left[\int_{T} \phi_{t}\left(f\left(x_{t}\right)\right) d \mu(t), g\left(\int_{T} \phi_{t}\left(x_{t}\right) d \mu(t)\right)\right] & \\
& \geq \inf _{m \leq z \leq M} F[f(z), g(z)] \mathbf{1} .
\end{aligned}
$$

In the dual case (when $f$ is operator concave) the opposite inequality holds with sup instead of inf.

We also give generalizations of some results from [5].

THEOREM 3.5. Let $f$ be a convex function on $[0, \infty)$ and let $\|\cdot\|$ be a normalized unitarily invariant norm on $B(H)$ for some finite dimensional Hilbert space $H$. Let $\left(\phi_{t}\right)_{t \in T}$ be a unital field of positive linear maps $\phi_{t}: B(H) \rightarrow$ $B(K)$, where $K$ is a Hilbert space, defined on a locally compact Hausdorff space $T$ equipped with a Radon measure $\mu$. Then for every continuous field of 
positive operators $\left(A_{t}\right)_{t \in T}$ we have

$$
\int_{T} \phi_{t}\left(f\left(A_{t}\right)\right) d \mu(t) \leq f(0) \mathbf{1}+\int_{T} \frac{f\left(\left\|A_{t}\right\|\right)-f(0)}{\left\|A_{t}\right\|} \phi_{t}\left(A_{t}\right) d \mu(t) .
$$

Especially, for $f(0) \leq 0$, the inequality

$$
\int_{T} \phi_{t}\left(f\left(A_{t}\right)\right) d \mu(t) \leq \int_{T} \frac{f\left(\left\|A_{t}\right\|\right)}{\left\|A_{t}\right\|} \phi_{t}\left(A_{t}\right) d \mu(t) .
$$

is valid.

Proof. Since $f$ is a convex function, $f(x) \leq \frac{M-x}{M-m} f(m)+\frac{x-m}{M-m} f(M)$ for every $x \in[m, M]$ where $m \leq M$. Since $\|\cdot\|$ is normalized and unitarily invariant, we have $0<A_{t} \leq\left\|A_{t}\right\| \mathbf{1}$ and thus

$$
f\left(A_{t}\right) \leq \frac{\left\|A_{t}\right\| \mathbf{1}-A_{t}}{\left\|A_{t}\right\|} f(0)+\frac{A_{t}}{\left\|A_{t}\right\|} f\left(\left\|A_{t}\right\|\right)
$$

for every $t \in T$. Applying positive linear maps and integrating we obtain

$$
\begin{aligned}
& \int_{T} \phi_{t}\left(f\left(A_{t}\right)\right) d \mu(t) \\
& \leq f(0)\left[\mathbf{1}-\int_{T} \frac{\phi_{t}\left(A_{t}\right)}{\left\|A_{t}\right\|} d \mu(t)\right]+\int_{T} \frac{f\left(\left\|A_{t}\right\|\right)}{\left\|A_{t}\right\|} \phi_{t}\left(A_{t}\right) d \mu(t)
\end{aligned}
$$

or

$$
\int_{T} \phi_{t}\left(f\left(A_{t}\right)\right) d \mu(t) \leq f(0) \mathbf{1}+\int_{T} \frac{f\left(\left\|A_{t}\right\|\right)-f(0)}{\left\|A_{t}\right\|} \phi_{t}\left(A_{t}\right) d \mu(t) .
$$

Note that since $\int_{T} \frac{\phi_{t}\left(A_{t}\right)}{\left\|A_{t}\right\|} d \mu(t) \leq \int_{T} \frac{\left\|A_{t}\right\| \phi_{t}(\mathbf{1})}{\left\|A_{t}\right\|} d \mu(t)=\mathbf{1}$ we obtain, for $f(0) \leq$ 0 , inequality (14) from (15).

Remark 3.6. Setting $T=\{1\}$ the inequality (14) gives

$$
\phi(f(A)) \leq \frac{f(\|A\|)}{\|A\|} \phi(A) .
$$

Furthermore, setting $\phi=1$, we get the inequality $f(\|A\|) \geq\|f(A)\|$ obtained in [5] under the assumption that $f$ is a nonnegative convex function with $f(0)=0$.

Related inequalities may be obtained by using subdifferentials. If $f: \mathbf{R} \rightarrow \mathbf{R}$ is a convex function and $[m, M]$ is a closed bounded real interval, then a 
subdifferential function of $f$ on $[m, M]$ is any function $k:[m, M] \rightarrow \mathbf{R}$ such that

$$
k(x) \in\left[f_{-}^{\prime}(x), f_{+}^{\prime}(x)\right], \quad x \in(m, M),
$$

where $f_{-}^{\prime}$ and $f_{+}^{\prime}$ are the one-sided derivatives of $f$ and $k(m)=f_{+}^{\prime}(m)$ and $k(M)=f_{-}^{\prime}(M)$. Since these functions are Borel measurable, we may use the Borel functional calculus. Subdifferential function for concave functions is defined in analogous way.

THEOREM 3.7. Let $\left(x_{t}\right)_{t \in T}$ be a bounded continuous field of self-adjoint elements in a unital $C^{*}$-algebra $\mathscr{A}$ with spectra in $[m, M]$ defined on a locally compact Hausdorff space T equipped with a Radon measure $\mu$, and let $\left(\phi_{t}\right)_{t \in T}$ be a unital field of positive linear maps $\phi_{t}: \mathscr{A} \rightarrow \mathscr{B}$ from $\mathscr{A}$ to another unital $C^{*}$-algebra $\mathscr{B}$. If $f: \mathbf{R} \rightarrow \mathbf{R}$ is a convex function then

$$
\begin{aligned}
f(y) \mathbf{1} & +k(y)\left(\int_{T} \phi_{t}\left(x_{t}\right) d \mu(t)-y \mathbf{1}\right) \\
\leq & \int_{T} \phi_{t}\left(f\left(x_{t}\right)\right) d \mu(t) \\
\leq & f(x) \mathbf{1}-x \int_{T} \phi_{t}\left(k\left(x_{t}\right)\right) d \mu(t)+\int_{T} \phi_{t}\left(k\left(x_{t}\right) x_{t}\right) d \mu(t)
\end{aligned}
$$

for every $x, y \in[m, M]$, where $k$ is a subdifferential function of $f$ on $[m, M]$. In the dual case ( $f$ is concave) the opposite inequality holds.

Proof. Since $f$ is convex we have $f(x) \geq f(y)+k(y)(x-y)$ for every $x, y \in[m, M]$. By using the functional calculus it then follows that $f\left(x_{t}\right) \geq$ $f(y) \mathbf{1}+k(y)\left(x_{t}-y \mathbf{1}\right)$ for $t \in T$. Applying the positive linear maps $\phi_{t}$ and integrating, LHS of (17) follows. The RHS of (17) follows similarly by using the functional calculus in the variable $y$.

Numerous inequalities can be obtained from (17). For example, LHS of (17) may be used to obtain an estimation from below in the sense of Theorem 3.1. Namely, the following theorem holds.

THEOREM 3.8. Let $\left(x_{t}\right)_{t \in T}$ be a bounded continuous field of self-adjoint elements in a unital $C^{*}$-algebra $\mathscr{A}$ with spectra in $[m, M]$ defined on a locally compact Hausdorff space T equipped with a Radon measure $\mu$, and let $\left(\phi_{t}\right)_{t \in T}$ be a unital field of positive linear maps $\phi_{t}: \mathscr{A} \rightarrow \mathscr{B}$ from $\mathscr{A}$ to another unital $C^{*}$-algebra $\mathscr{B}$. Let $f: \mathbf{R} \rightarrow \mathbf{R}, g:[m, M] \rightarrow \mathbf{R}$ and $F: U \times V \rightarrow \mathbf{R}$ be functions such that $f([m, M]) \subset U, g([m, M]) \subset V, F$ is bounded, $f$ is convex and $f(y)+k(y)(t-y) \in U$ for every $y, t \in[m, M]$, where $k$ is a subdifferential function of $f$ on $[m, M]$. If $F$ is operator monotone in the first 
variable, then

$$
\begin{aligned}
F\left[\int_{T} \phi_{t}\left(f\left(x_{t}\right)\right) d \mu(t), g\right. & \left.\left(\int_{T} \phi_{t}\left(x_{t}\right) d \mu(t)\right)\right] \\
& \geq \inf _{m \leq z \leq M} F[f(y)+k(y)(z-y), g(z)] \mathbf{1}
\end{aligned}
$$

for every $y \in[m, M]$. In the dual case (when $f$ is concave) the opposite inequality holds in (18) with sup instead of inf.

Using LHS of (17) we can give generalizations of some dual results from [5].

THEOREM 3.9. Let $\left(x_{t}\right)_{t \in T}$ be a bounded continuous field of positive elements in a unital $C^{*}$-algebra $\mathscr{A}$ defined on a locally compact Hausdorff space $T$ equipped with a Radon measure $\mu$, and let $\left(\phi_{t}\right)_{t \in T}$ be a unital field of positive linear maps $\phi_{t}: \mathscr{A} \rightarrow \mathscr{B}$ from $\mathscr{A}$ to another unital $C^{*}$-algebra $\mathscr{B}$ acting on a finite dimensional Hilbert space $K$. Let $\|\cdot\|$ be a unitarily invariant norm on $B(K)$ and let $f:[0, \infty) \rightarrow \mathrm{R}$ be an increasing function.

(1) If $\|\mathbf{1}\|=1$ and $f$ is convex with $f(0) \leq 0$ then

$$
f\left(\left\|\int_{T} \phi_{t}\left(x_{t}\right) d \mu(t)\right\|\right) \leq\left\|\int_{T} \phi_{t}\left(f\left(x_{t}\right)\right) d \mu(t)\right\| .
$$

(2) If $\int_{T} \phi_{t}\left(x_{t}\right) d \mu(t) \leq\left\|\int_{T} \phi_{t}\left(x_{t}\right) d \mu(t)\right\| \mathbf{1}$ and $f$ is concave then

$$
\int_{T} \phi_{t}\left(f\left(x_{t}\right)\right) d \mu(t) \leq f\left(\left\|\int_{T} \phi_{t}\left(x_{t}\right) d \mu(t)\right\|\right) \mathbf{1} .
$$

Proof. Since $f(0) \leq 0$ and $f$ is increasing we have $k(y) y-f(y) \geq 0$ and $k(y) \geq 0$. From (17) and the triangle inequality we have

$$
k(y)\left\|\int_{T} \phi_{t}\left(x_{t}\right) d \mu(t)\right\| \leq\left\|\int_{T} \phi_{t}\left(f\left(x_{t}\right)\right)\right\|+(k(y) y-f(y)) .
$$

Now (19) follows by setting $y=\left\|\int_{T} \phi_{t}\left(x_{t}\right) d \mu(t)\right\|$. Inequality (20) follows immediately from the assumptions and from the dual case of LHS in (17) by setting $y=\left\|\int_{T} \phi_{t}\left(x_{t}\right) d \mu(t)\right\|$.

Finally, to illustrate how RHS of (17) works, we set

$$
x=\frac{\left\|\int_{T} \phi_{t}\left(k\left(x_{t}\right) x_{t}\right) d \mu(t)\right\|}{\left\|\int_{T} \phi_{t}\left(k\left(x_{t}\right)\right) d \mu(t)\right\|}
$$


and obtain a Slater type inequality

$$
\int_{T} \phi_{t}\left(f\left(x_{t}\right)\right) d \mu(t) \leq f\left(\frac{\left\|\int_{T} \phi_{t}\left(k\left(x_{t}\right) x_{t}\right) d \mu(t)\right\|}{\left\|\int_{T} \phi_{t}\left(k\left(x_{t}\right)\right) d \mu(t)\right\|}\right) \mathbf{1}
$$

under the condition

$$
\frac{\int_{T} \phi_{t}\left(k\left(x_{t}\right) x_{t}\right) d \mu(t)}{\left\|\int_{T} \phi_{t}\left(k\left(x_{t}\right) x_{t}\right) d \mu(t)\right\|} \leq \frac{\int_{T} \phi_{t}\left(k\left(x_{t}\right)\right) d \mu(t)}{\left\|\int_{T} \phi_{t}\left(k\left(x_{t}\right)\right) d \mu(t)\right\|} .
$$

\section{REFERENCES}

1. Aujla, J. S., Vasudeva, H. I., Operator inequalities related to means of operators, Math. Japon. 41 (1995), 383-388.

2. Bhatia, R., Matrix Analysis, Springer, Germany, 1996.

3. Choi, M. D., A Schwarz inequality for positive linear maps on $C^{*}$-algebras, Illinois J. Math. 18 (1974), 565-574.

4. Davis, Ch., A Schwarz inequality for convex operator functions, Proc. Amer. Math. Soc. 8 (1957), 42-44.

5. Drnovšek, R., Kosem, T., Inequalities between $f(\|A\|)$ and $\|f(|A|)\|$, Math. Inequal. Appl. 8 (2005), 1-6.

6. Hansen, F., Operator monotone functions of several variables, Math. Ineqal. Appl. 6 (2003), $1-17$.

7. Hansen, F., Pedersen, G. K., Jensen's inequality for operators and Löwner's theorem, Math. Ann. 258 (1982), 229-241.

8. Hansen, F., Pedersen, G. K., Jensen's operator inequality, Bull. London Math. Soc. 35 (2003), 553-564.

9. Fujii, M., Kubo, F., Around Jensen's inequality II, Math. Japon. 27 (1982), 495-499.

10. Furuta, T., Mićić-Hot, J., Pečarić, J., Seo, Y., Mond-Pečarić Method in Operator Inequalities, Element, Zagreb, 2005.

11. Kubo, F., Ando, T., Means of positive linear operators, Math. Ann. 246 (1980), 205-224.

12. Mond, B., Pečarić, J., Converses of Jensen's inequality for several operators, Rev. Anal. Numér. Théor. Approx. 23 (1994), 179-183.

13. Mond, B., Pečarić, J., On Jensen's inequality for operator convex functions, Houston J. Math. 21 (1995), 739-754.

14. Tominaga, M., The upper bound of a reverse Hölder's type operator inequality and its applications, J. Inequal. Appl. 7 (2002), 633-645.

DEPARTMENT OF ECONOMICS

COPENHAGEN UNIVERSITY

STUDIESTRAEDE 6

1455 COPENHAGEN K

DENMARK

E-mail: frank.hansen@econ.ku.dk
FACULTY OF TEXTILE TECHNOLOGY UNIVERSITY OF ZAGREB PIEROTTIJEVA 6

10000 ZAGREB

CROATIA

E-mail: pecaric@hazu.hr 
FACULTY OF CHEMICAL ENGINEERING AND TECHNOLOGY

UNIVERSITY OF ZAGREB

MARULIĆEV TRG 19

10000 ZAGREB

CROATIA

E-mail: iperic@pbf.hr 
